\title{
Free-standing graphene at atomic resolution
}

\author{
MHAIRI H. GASS ${ }^{1 \star \dagger}$, URSEL BANGERT ${ }^{2 \dagger}$, ANDREW L. BLELOCH ${ }^{1}$, PENG WANG'1, RAHUL R. NAIR²,3 \\ AND A. K. GEIM ${ }^{3}$ \\ ${ }^{1}$ SuperSTEM, STFC Daresbury Laboratory, Warrington WA4 4AD, UK \\ ${ }^{2}$ School of Materials, University of Manchester, Manchester M13 9PL, UK \\ ${ }^{3}$ Manchester Centre for Mesoscience and Nanotechnology, University of Manchester, Manchester M13 9PL, UK \\ tThese authors contributed equally to this work. \\ *e-mail: m.h.gass@liv.ac.uk
}

Published online: 28 September 2008; doi:10.1038/nnano.2008.280

Research interest in graphene, a two-dimensional crystal consisting of a single atomic plane of carbon atoms, has been driven by its extraordinary properties, including charge carriers that mimic ultra-relativistic elementary particles. Moreover, graphene exhibits ballistic electron transport on the submicrometre scale, even at room temperature, which has allowed the demonstration of graphene-based field-effect transistors and the observation of a room-temperature quantum Hall effect. Here we confirm the presence of free-standing, single-layer graphene with directly interpretable atomic-resolution imaging combined with the spatially resolved study of both the $\pi \rightarrow \pi^{*}$ transition and the $\pi+\sigma$ plasmon. We also present atomic-scale observations of the morphology of free-standing graphene and explore the role of microstructural peculiarities that affect the stability of the sheets. We also follow the evolution and interaction of point defects and suggest a mechanism by which they form ring defects.

Recent measurements of the remarkable electronic properties of graphene have resulted in intense research activity on twodimensional (2D) crystals $^{1-5}$. Unlike most materials in condensed matter physics, where the Schrödinger equation can be used to describe their electronic properties, for graphene the charge carriers mimic relativistic particles and can thus be described using the Dirac equation ${ }^{3}$.

The ability of extended 2D structures to exist is the subject of a long-standing theoretical debate, and it has previously been suggested that $2 \mathrm{D}$ films embedded in three-dimensional (3D) space can be stabilized by out-of-plane undulations ${ }^{6,7}$. Elucidating the atomic structure of graphene may seem blindingly obvious at first consideration, but, given that it is necessarily an 'imperfect' 2D crystal, it offers insight in three important ways. First, direct imaging of atoms combined with energy-loss spectroscopy provides further corroboration of the existence of areas of free-standing monolayers of carbon atoms. Second, revealing the atomic structure of the edges of graphene and the fundamental topological defects within adds insight to the stability issues, as does the characterization of the surface contamination believed to consist mainly of hydrocarbons ubiquitously found on graphene. This last point may also provide clues as to certain limitations in the electronic behaviour of graphene films.

Evidence of the existence of free-standing graphene has been obtained from electron diffraction experiments ${ }^{3}$, which, in this case, was averaged over approximately a square micrometre of material. Recently, others have presented defect configurations in suspended graphene using bright-field phase contrast ${ }^{8}$. The appearance of atomic structure in phase contrast in the case of $3 \mathrm{D}$ crystals is not immediately interpretable, and even in $2 \mathrm{D}$ crystals, is sensitive to focusing conditions. However, the atomic lattice seen in high-angle annular dark-field (HAADF) images acquired in a scanning transmission electron microscope
(STEM), with the electron beam focused onto a mono-atomic layer of atoms, such as graphene, are directly interpretable; they are a direct depiction of the ball-and-stick model of an atomic lattice structure, where bright contrast corresponds to atoms and dark contrast to the gaps between. The effects of atomic structure extending in the direction of the electron beam, resulting in dechannelling of the beam on atomic columns and hence inducing contrast changes and so on, cannot occur in monolayer structures. Moreover, the arrangements of atomic-scale defects can be deduced in a straightforward manner. Furthermore, single graphene sheets exhibit unique behaviour in their electron energy-loss spectra, and this can be conveniently accessed by highly spatially resolved electron energy-loss spectroscopy (EELS $)^{9}$. The spectroscopy needs to be localized mainly because surface contamination exists on all graphene other than on small patches, as observed here, leaving only small patches of genuinely monoatomic graphene.

The low-loss EELS spectrum of graphitic structures is dominated by plasmon excitations consisting of $\pi$ - and $\sigma$-plasmons, both exhibiting bulk and surface modes. The shape of the low-loss EELS spectrum for graphitic structures is highly dependent on the angle at which the incident beam hits the structure. Nanotubes are an excellent example of this, as demonstrated both theoretically and experimentally ${ }^{10,11}$. For example, in multiwalled carbon nanotubes, a spectrum from perpendicular impact will show a plasmon excitation at $27 \mathrm{eV}$, whereas impact parallel to the graphene sheet exhibits a plasmon excitation at $23 \mathrm{eV}$ (ref. 11). A trend has been observed for the $\pi+\sigma$ plasmon in single-walled nanotubes ${ }^{10}$, namely that the tangential (or in-plane) mode increases at the expense of the radial (or out-of-plane) mode when the radius increases, and for $r \rightarrow \infty$ it has been predicted that only the tangential mode (at $\sim 15 \mathrm{eV}$ ) remains. A word of caution has to be mentioned here: previous nanotube calculations assume an aloof beam, but 
a

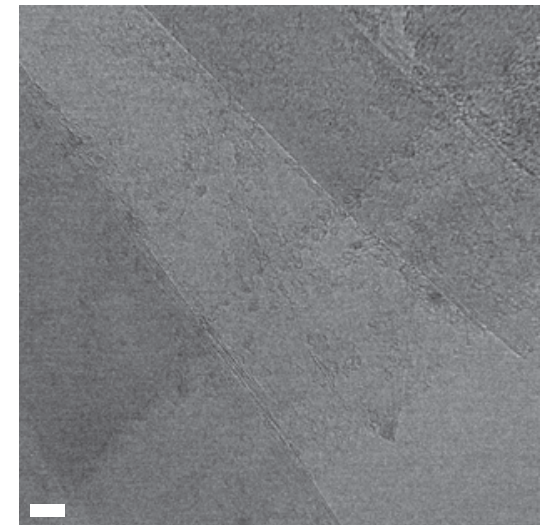

$\boldsymbol{6}$

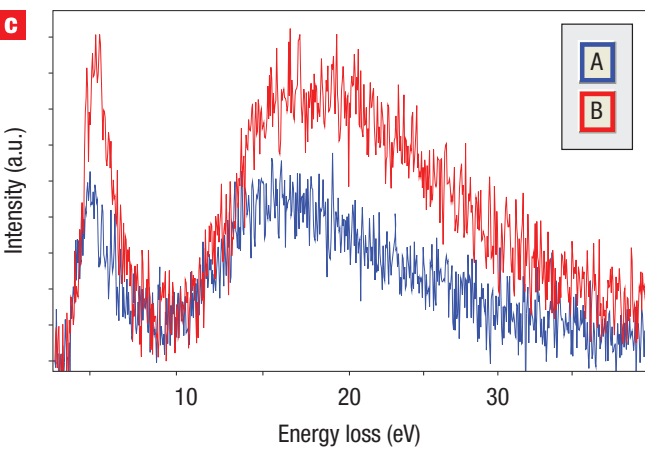

b

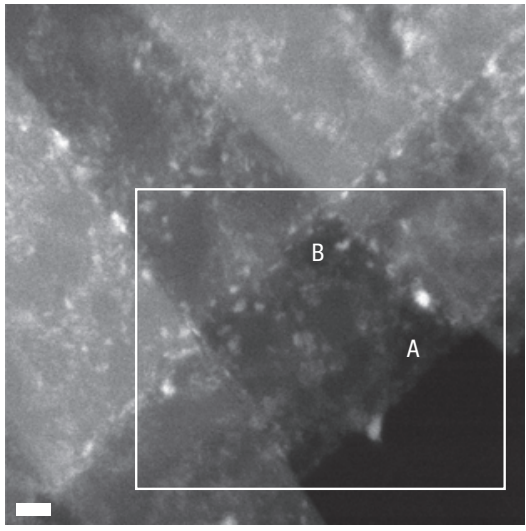

d

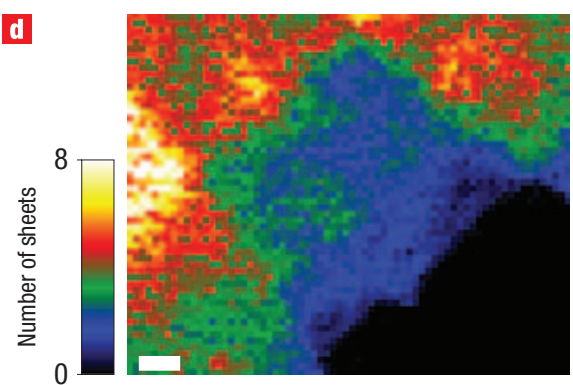

Figure 1 Thickness analysis of graphene layers. $\mathbf{a}, \mathbf{b}$, Bright-field (a) and HAADF (b) images of an area of sample with a varying number of graphene layers. c, An EELS spectrum image was acquired from the boxed area in $\mathbf{b}$ and spectra extracted from the areas indicated by $A$ (blue trace) and B (red trace) for comparison. d, Thickness map created from the EELS spectrum image for the region inside the white rectangle in $\mathbf{b}$, with the thinnest area $A$ assumed to be a single layer of graphene. All scale bars, $5 \mathrm{~nm}$.

Table 1 HAADF and EELS values obtained from analysed areas in Figs 1 and 2. EELS values (not in parentheses) were averaged over six pixels, except for the vacuum values, which were averaged over 100 pixels. Values in parentheses were averaged over 25 pixels. HAADF values (not in parentheses) were averaged over 616 pixels, except for the vacuum values, which were averaged over $\mathbf{4 0 0}$ pixels (the number of pixels used for averaging in the HAADF image is greater than the EELS map due to the greater pixel density in the HAADF image). HAADF values in parentheses were averaged over varying pixel numbers, all larger than 400.

\begin{tabular}{lllll}
\hline & Single-layer & Two-layer & Five-layer & Vacuum \\
\hline HAADF (relative intensity) & $1 \pm 0.82(1 \pm 0.43)$ & $2.7 \pm 1.07(1.86 \pm 0.86)$ & $(5 \pm 0.43)$ & $0.01 \pm 0.53(0 \pm 0.3)$ \\
EELS (relative intensity) & (A), $1 \pm 0.09(1 \pm 0.21)$ & $(\mathrm{B}), 2.3 \pm 0.18(1.89 \pm 0.14)$ & $(4.73 \pm 0.16)$ & $0.02 \pm 0.14(0.01 \pm 0.04)$ \\
\hline
\end{tabular}

in our case the beam penetrates the graphene sheet. We have recently carried out $a b$ initio calculations of the low-loss function for graphene with impact perpendicular to the sheet, where the potential takes electron transit within the foil into account, and compared them with experiment ${ }^{9}$. The theoretical predictions for $\pi$ - and $\pi+\sigma$-surface plasmon modes in free-standing single sheets give $\sim 4.5$ and $\sim 14.5 \mathrm{eV}$, which are substantially redshifted from their values in graphite, in excellent agreement with the experimental spectra, as will be shown below. The calculations also predict there is little loss caused by out-of-plane modes for energies less than $\sim 10 \mathrm{eV}$.

\section{DIRECT CONFIRMATION OF THE GRAPHENE LATTICE}

Figure 1a,b shows medium-resolution bright-field and HAADF images of the graphene sample acquired simultaneously using STEM. HAADF imaging is of particular use for imaging varying numbers of graphene sheets as its intensity varies monotonically with sample thickness for thin samples ${ }^{12}$; the change in contrast in Fig. 1b suggests that the number of graphene layers varies over the area. A low-loss EELS spectrum image was acquired over the boxed area indicated in Fig. 1b and spectra extracted from two of the thinnest areas for comparison (Fig. 1c). Features of interest, namely the slight downshift in energy of the $\pi \rightarrow \pi^{*}$ transition between spectra B and A, can be noted. Both of these values are considerably lower in energy than the $\pi \rightarrow \pi^{*}$ transition for graphite of $6.2 \mathrm{eV}$ (ref. 13). Further, the shape of the $\pi+\sigma$ plasmon for spectrum A suggests that there is almost no bulk component with the peak occurring at $\sim 15 \mathrm{eV}$, but spectrum $\mathrm{B}$ has a small contribution from the bulk plasmon, resulting in a higher peak energy. Again, this can be compared to the plasmon features from graphite where the $\pi+\sigma$ plasmon peak occurs at $26 \mathrm{eV}$ (ref. 13). It is inherently difficult to acquire data from a perfectly clean area of graphene, as even the cleanest patches will attract a thin surface layer of contamination during analysis, resulting in a slightly higher plasmon energy than 

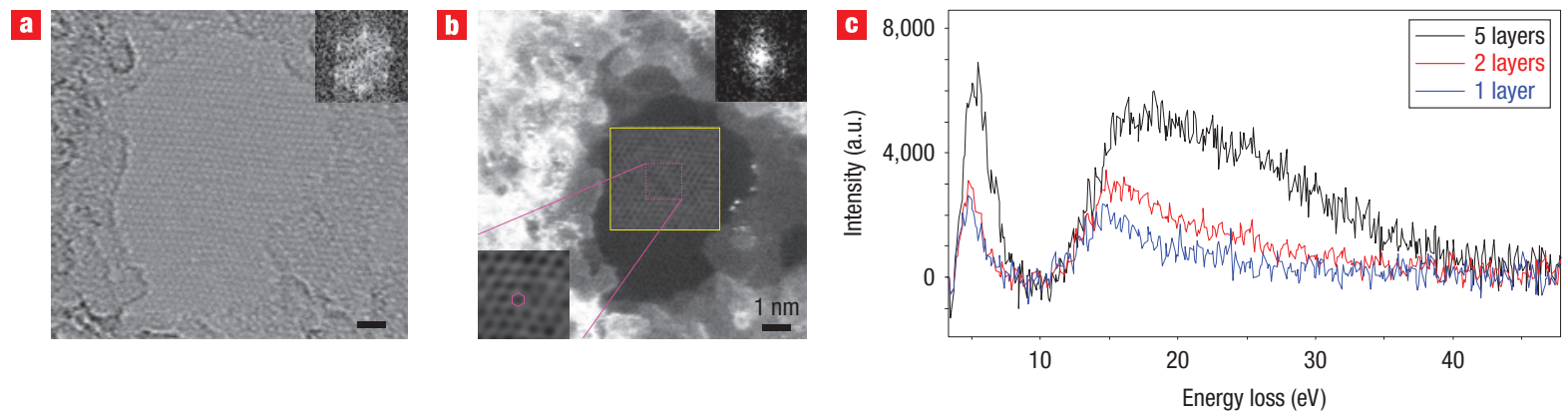

Figure 2 High-resolution images of mono-layer graphene. a,b, Bright-field (a) and HAADF (b) images of the monolayer, showing a clean patch of graphene surrounded by a mono-atomic surface layer; individual contaminant atoms of higher atomic number can be seen in $\mathbf{b}$. The inset FFT clearly shows the lattice in the HAADF image and, by applying a bandpass filter, the atomic structure is apparent. c. EELS spectra were acquired from the clean area; a background-subtracted spectrum summed over 25 pixels is shown as a purple trace. For direct comparison unscaled spectra acquired for two-layer and five-layer graphene from nearby areas, also summed over 25 pixels, are also shown as orange and black traces, respectively. Scale bars in a and $\mathbf{b}, 1 \mathrm{~nm}$.

predicted. Taking the layer measured in A to be a single graphene layer, a relative thickness map was calculated from the EELS spectrum image (Fig. 1d) by integrating the EELS intensity between 10 and $40 \mathrm{eV}$ in each spectrum. The number of layers measured from points $\mathrm{A}$ and $\mathrm{B}$ and from vacuum are shown in Table 1; relative values from the HAADF image were also calculated from the same points for comparison. It can be seen from both the HAADF image (Fig. 1b) and the EELS thickness map that the layers are not uniform in thickness; this is due to the surface contamination present on the sample, although area $\mathrm{A}$ is particularly clean. The measured number of layers from both the HAADF and EELS thicknesses are in good agreement and both suggest that if area $\mathrm{A}$ is a single graphene layer then area B consists of two graphene layers, possibly with a small amount of surface contamination. This also agrees with the EELS spectra. Because they have been acquired from the same spectrum image, the intensities of the EELS spectra are directly comparable and show that the plasmon from area $B$ is predominately surface plasmon with a small amount of bulk contribution.

High-resolution images were acquired from a single-layer area. The bright-field and HAADF images (Fig. 2a and b, respectively) clearly show a clean area of graphene surrounded by surface contamination, which presumably consists of hydrocarbon chains. The fast Fourier transform (FFT) is included on both images (top right inset), and overlaid on the HAADF image is an area of contrast to which a filter was applied (see Methods) with an enlarged part as an inset (bottom left). The $\mathrm{C}-\mathrm{C}$ bond length in graphite is $1.42 \AA$ and it is possible to see the graphene hexagons; one of these is marked in purple. In the HAADF images atoms are seen as bright features, and these constitute the corners of the hexagon. The $\mathrm{C}-\mathrm{C}$ bond length, although not clearly resolved everywhere in the patch, is measured to be $1.4 \AA$ and the diameter across the hexagons, atom to atom, is $2.8 \AA$, confirming that this is a single layer. Low-loss EELS was acquired from this clean monolayer patch. Figure $2 \mathrm{c}$ shows the resulting spectrum, which is compared to two other spectra taken from two- and five-layer regions nearby. Optical microscopy was further used to confirm the number of layers through analysis of the transmitted colour ${ }^{14}$, as well as by measuring the HAADF and EELS intensities as previously described. The values of the HAADF and the integrated EELS intensity are shown in parentheses in Table 1. The results in Fig. 2 are from a different sample to that in Fig. 1, and were also taken on a different day; although the table values differ in their absolute value, the increments give precisely the same trend. In Fig. $2 \mathrm{c}$ the $\pi$ plasmon notably shifts to $4.7 \mathrm{eV}$ in the single sheet and the $\pi+\sigma$ plasmon consists only of the surface mode at $14.5 \mathrm{eV}$. We note that these values are in exact agreement with those of free-standing graphene predicted by theory ${ }^{9}$. We also note that it is only in pristine patches like in Fig. $2 \mathrm{c}$ that this is the case; spreading of the contaminant surface layer over the clean graphene patch, as often happens during analysis, immediately adds an out-of-plane component to the $\pi+\sigma$ plasmon, resulting in an apparent peak shift, and a general increase in intensity above $14.5 \mathrm{eV}$. This explains the slightly higher values for the $\pi+\sigma$ plasmon energy measured for the single layer in Fig. 1.

\section{DEFECTS IN GRAPHENE}

We now concentrate on the atomic structure within clean graphene patches. There is a wealth of literature dealing with defects in highly ordered pyrolytic (HOP) graphite ${ }^{15-18}$. Isolated point defects on the surface of graphite are exclusively reported in connection with irradiation damage or ion bombardment; this seems to indicate that even the defects seen in the very first STEM scan occur due to electron bombardment.

The vast majority of defects in our HAADF images are single vacancies. Figure 3 shows two constellations of single-vacancy defects - a mono- and a di-vacancy (with overlaid ball-and-stick model)_but other constellations, for example, two single vacancies bridged by a C-atom, are also frequently seen in atomic-resolution HAADF images. Vacancy constellations are easy to discern, whereas Stone-Wales defects ${ }^{19}$, constituting a reconfigured, rotated bond, have not so far been identified.

We also observe a second type of defect, related to the termination of a row of hexagons, and hence representing the $2 \mathrm{D}$ or monolayer equivalent of a dislocation. This defect structure is quite common, and examples are depicted in Fig. 4a,b. The sketch in Fig. 4c shows how a structure consisting of two dissociated defects, each consisting of a double pentagon (8-atom ring), could be produced by rearrangement of atoms that were originally part of a 4-vacancy defect constellation. The latter consists of four groups, each comprising three hexagons joined by a vacancy at the centre, as in the model in Fig. 4c (left). We have observed closely spaced vacancies as suggested in this sketch. Reconstruction results in formation of two 8-atom defects joined by a 6-ring at their 'convex' side, between them (Fig. 4c, middle). This keeps the total number of atoms in the cluster constant, while using up one of the 6-rings. There is therefore 


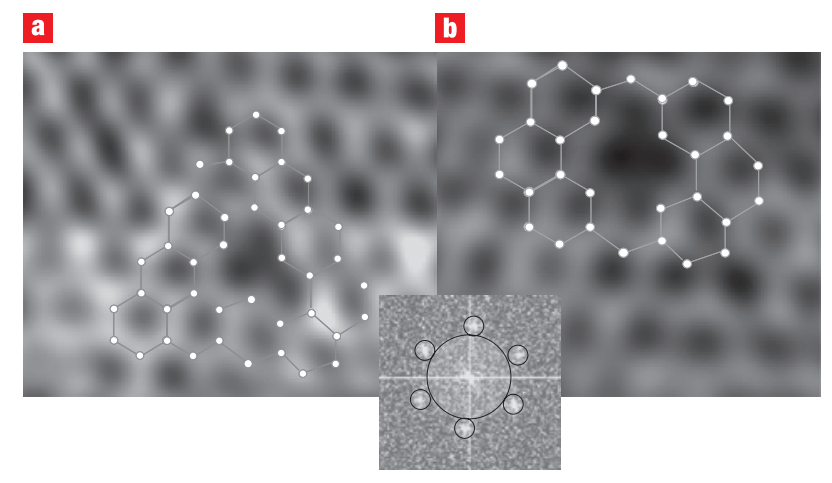

Figure 3 HAADF lattice images of defects. a,b, The images were obtained with a filter applied to the FFT of the raw images as indicated by the inset and show a mono-vacancy (a) and a di-vacancy (b).

only one 6-atom ring between the defects, but two 6-atom rings joining the 'outside' of the defects. Subsequent rearrangement of bonds can result in further separation along the row of hexagons marked by the grey line (Fig. $4 \mathrm{c}$, right). The separation can thus become large. Because the reconstruction required to form the two 8-atom defects proceeds under consumption of one 6-atom ring, there will be a difference of one row of hexagons joining the defect on the 'convex inside' and the 'concave outside', hence the termination of one row of hexagons on the concave side. The termination of near parallel rows is indicated in Fig. 4a,b (in Fig. 4a we also mark the termination of diagonal rows). In Fig. 4a the defects are only separated by two hexagons along the grey line; in Fig. $4 \mathrm{~b}$ they are separated by a larger number. Because the defect movement does not require vacancies, it would be similar to dislocation glide. However, in order to create the defect from the original 4-vacancy constellation in the first place, reconstruction of four bonds is required; this is a highly energetic process, and although vacancy movement under the electron beam is readily observed, in the absence of any apparent external driving force it might still be deemed unlikely. However, 8 -atom rings can also occur through reconstruction of the edge of a graphene layer, which exhibits a distortion, as shown in Fig. 4d (see row of sheared hexagons). Here an 8-atom ring defect bridges a corner. The presence of residual shear stresses (for example, originating from micromechanical cleaving and exfoliation of the graphene) is a sufficient source to initiate glide of defects resulting from graphene sheet-edge reconstruction from opposite sides of the graphene flake. In this case no vacancies would be required, and each glide step (that is, moving the defect by one hexagon along the grey line) only requires reconstruction of one bond.

Intensity changes occurring from one atom to the next and often 'highlighting' several consecutive atoms in a chain-like fashion can notably be seen in all graphene HAADF images. The contrast variations are not random enough to arise from probe noise or from position instability of the probe or the atoms due to lattice vibrations. It could be speculated that intensity changes point to the existence of adsorbed species, such as $\mathrm{H}$ or $\mathrm{O}$. $\mathrm{H}$ adatoms would have to be ruled out, as these would lead to dramatic changes in electronic properties; however, the variations in intensity of atoms cannot arise from any element heavier than H. Molecular hydrogen is present in ample quantities in all UHV systems, so absorption of $\mathrm{H}_{2}$ might be a possibility. Typical intensity ratios measured for brighter and less bright atoms, as for example indicated by arrows in Fig. 4 (vacuum value subtracted),
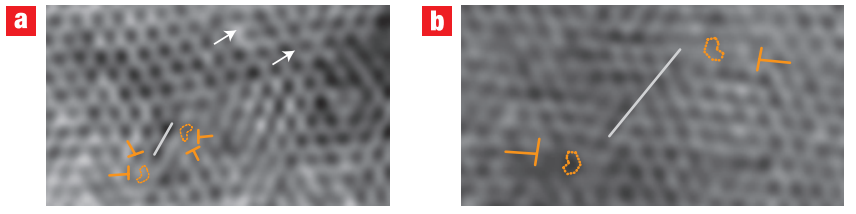

G

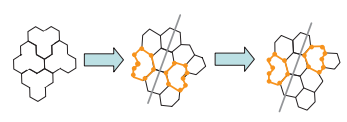

d

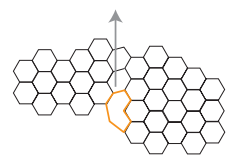

Figure 4 Termination of hexagon rows in HAADF images.

$\mathbf{a}, \mathbf{b}$, The termination of hexagon rows is indicated in orange, near defects with small (a) and wide (b) separation along the direction marked by the grey lines. c, Model for creation of two ring defects from a 4-vacancy constellation involving 34 atoms with consumption of one hexagon, which leads to three hexagons joining on the concave, and two hexagons on the convex side of the defects. The defects can glide apart along the grey line by rearranging just one bond in each unit-cell glide step. $\mathbf{d}$, Formation of the same defect by bond reconstruction at the edge of a graphene sheet with a distortion; glide inwards along the grey arrow might be achieved by sheer stress, leading, as in $\mathbf{c}$, to termination of an extra row on the convex side of the defect. Arrows in a mark atoms with different intensities.

are 36:39, whereas theoretically, using the $Z^{2}$ dependence, the intensity change from $\mathrm{C}$ to $\mathrm{C}$ with $\mathrm{H}_{2}$ above/below would be predicted as $\sim 36: 38$. This is in remarkably good agreement with the measurement. Note that the intensity measurements were made on unfiltered (that is, raw) HAADF images, and the vacuum intensity level was taken into account.

\section{EDGE RECONSTRUCTIONS IN GRAPHENE}

Finally we touch on an unusual edge reconstruction. Associated with the edges of the foil or changes in thickness of the foil, in a significant number of cases, we observe two lines separated by $0.4 \mathrm{~nm}$. These can be either (i) staggered edges, (ii) a double layer folded back on itself, representing four layers, or (iii) the smallest possible scroll, resembling a small diameter single-walled nanotube indicated by the diagram in Fig. 5. The edge reconstruction of graphitic-type structures has previously been studied $^{20}$ and nanotube-like surface structures observed ${ }^{21}$. Here we argue that the edge can be represented by case (iii). Observations of graphene sheet edges are shown in Fig. $5 \mathrm{a}-\mathrm{c}$. In particular, Fig. $5 \mathrm{~b}$ shows the edge of a free-standing graphene layer, which has rolled up into a nanotube-like structure; second and third layers are also present (indicated by arrows), their edges having also rolled up into scroll-like structures. Figure 5a also shows individual sheets curling up at the end. An intensity profile from the respective HAADF image has been superimposed, showing that the thickness of the sample increases over these edges, and confirming the scroll-like structure rather than the folded sheet as schematically shown in Fig. $5 \mathrm{~d}$ (ii). Importantly, in the latter case the HAADF profile would not show an intensity increase between the double lines at the sheet edges. Further evidence for the scroll-like edge comes from simulations for both the bright-field and HAADF images in Fig. 5e. It is not yet clear how the scroll negotiates its bonding with the graphene; presumably its edge is held in place simply by van der Waals bonding with atoms in the underlying graphene, and thus it would constitute a nanotube-like structure with the smallest possible diameter. Figure $5 \mathrm{c}$ shows areas where several 
a

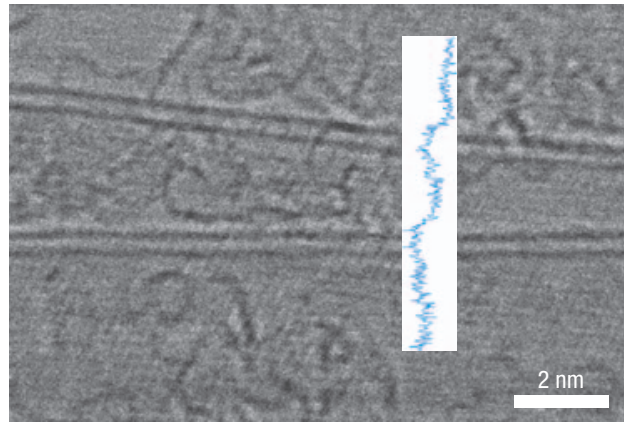

b

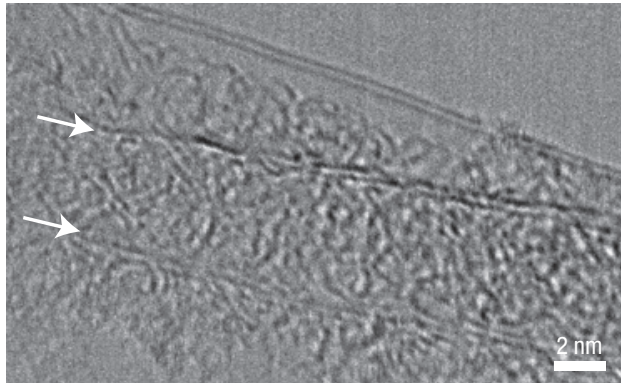

$\mathbf{G}$

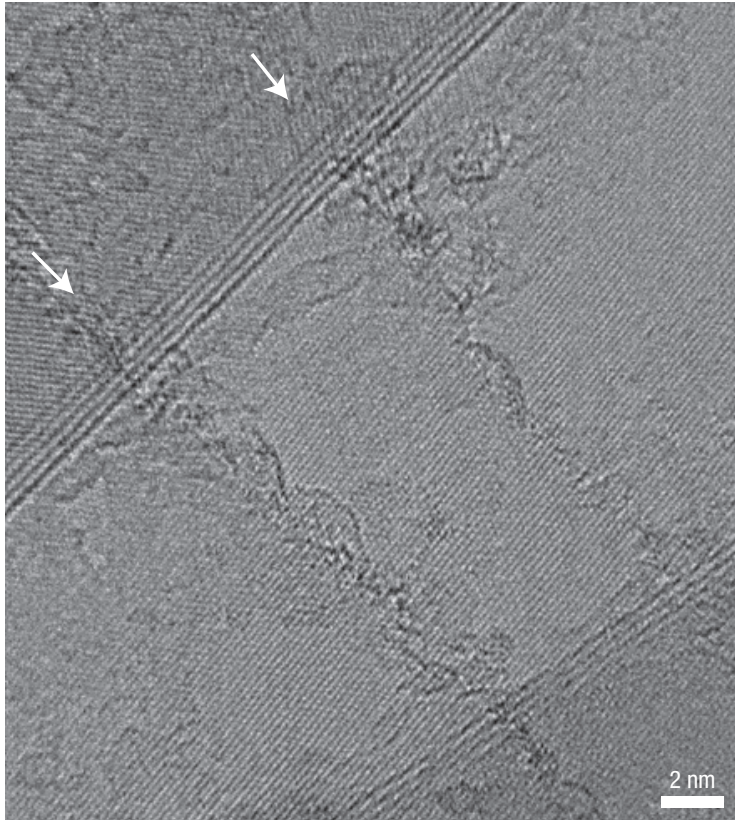

d

(i)

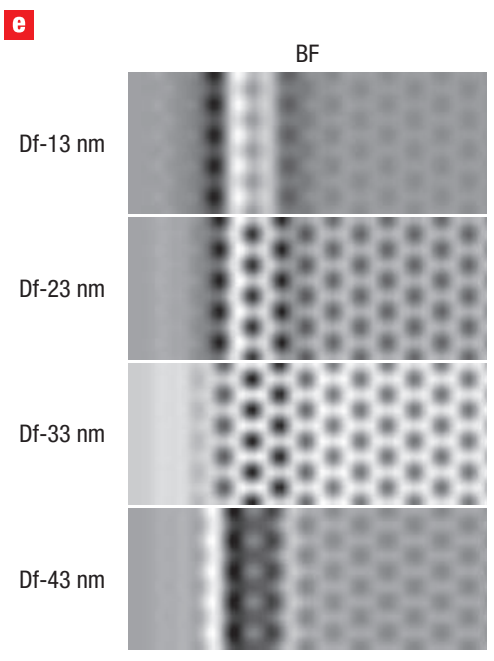

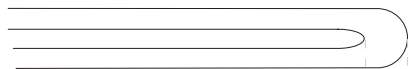

(ii) (iii)

Single sheet + one row C-hexagon

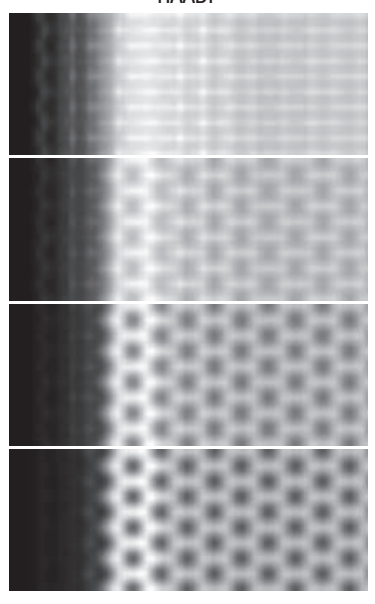

Figure $\mathbf{5}$ Edge structures of graphene. $\mathbf{a}-\mathbf{c}$, Bright-field images showing edges of graphene. Both $\mathbf{a}$ and $\mathbf{b}$ show the edge of the graphene layers forming the smallest possible scroll with a diameter of $0.4 \mathrm{~nm}$. The intensity profile from a HAADF image, taken simultaneously (not shown), is superimposed onto a, showing the increase in intensity over these edges. c, Multiple graphene sheets folded over together are visible, as well as single-layer edges running perpendicular to the folds (indicated by white arrows), which appear to be held down by surface contamination. Scale bars, $2 \mathrm{~nm}$. d, Cases where two lines are separated by $0.4 \mathrm{~nm}$ could be caused by the following situations: (i) staggered edges, (ii) a double layer folded back on itself, representing four layers or (iii) the smallest possible scroll, resembling a nanotube. e, Both the BF and HAADF images are simulated for the structure in case (iii) above (also shown in the atomistic model on the right) using Kirkland's code for a range of defoci and a spherical aberration of $50 \mu \mathrm{m}$, confirming that the edge reconstruction appears to be in the form of a scroll.

layers have rolled up together at the edges; an example of this is also shown in the inset of Fig. 1b. Sheets with edges running perpendicular do not roll up at the edge. This is likely to be due to one of three reasons: (i) the sheets in question are pinned down by nearby layers that are on top; (ii) there is surface contamination that has collected at the edges, pinning them down, which is clear in the HAADF image in Fig. 1b and has been observed several times; (iii) edge terminations in certain 
crystallographic directions may be more stable than others, and therefore may remain flat.

\section{CONCLUSIONS}

It has been shown that it is possible to directly image, at atomic resolution, individual free-standing graphene layers. The presence of graphene is confirmed by low-loss EELS where the energy of the $\pi$ and the $\pi+\sigma$ plasmons are significantly redshifted compared to two or more layers; measurements of the intensities of both HAADF images and plasmon losses in EELS also corroborate the presence of graphene. It is noted, however, that the slightest amount of surface contamination will alter the observed low-loss EELS structure. All detailed observations of graphene appear to show the presence of contamination, indicating that electrical measurements carried out on graphene are also on contaminated layers, even though this does not seem to significantly affect the electronic properties. It remains unclear whether the presence of the surface contamination, likely to be hydrocarbons, plays a part in the stabilization of the $2 \mathrm{D}$ structure, causing, or being associated with, small deformations of the film, or whether its presence is entirely due to the highly lipophilic nature of the graphene. Vacancy-type defects due to electron beam damage are directly observed, as well as a ring-type defect, which parallels a dislocation core in 3D structures, resulting from the glide movement of reconstructed atom arrangements at the edges of graphene sheets. It is also observed that where the end of the sheet is clean and straight enough, the edge rolls up into a nano-scroll of the diameter of the smallest possible nanotube.

\section{METHODS}

\section{SAMPLE PREPARATION}

Large graphene membranes were prepared by micromechanical cleavage ${ }^{22}$ of natural graphite on top of an oxidized silicon wafer. This deposition technique has the benefit of allowing a quick and easy identification of mono- and multiplelayer graphene by the optical contrast that occurs with respect to the oxidized wafer. By using photolithography, a perforated copper/gold film was deposited on top of the graphene crystallites, which could act as a scaffold to be used in standard transmission electron microscopy holders. This scaffold was lifted off the silicon wafer, leaving the graphene attached to it ${ }^{14}$. Before electron microscopy, optical microscopy was used to identify regions of monolayers.

\section{EXPERIMENTAL PROCEDURES}

The microscopy was carried out at the SuperSTEM facility, Daresbury, UK on a Vacuum Generators HB501 STEM fitted with a Nion second-generation aberration corrector and a Gatan Enfina EELS spectrometer. The operating voltage was $100 \mathrm{kV}$. Low-loss EELS was carried out with a dispersion of $0.05 \mathrm{eV}$ per channel at an energy resolution (full width at half-maximum, FWHM, of the zero loss peak) of around $0.3 \mathrm{eV}$. The convergence semi-angle was $24 \mathrm{mrad}$ and the collection semi-angle was approximately $5 \mathrm{mrad}$.

\section{HIGH-ANGLE ANNULAR DARK-FIELD IMAGING (HAADF)}

For thin samples the HAADF signal has been shown to be mono-atomic with thickness ${ }^{12}$. Relative sample thicknesses were measured by removing the intensity attributed to the vacuum signal (this is always set to a positive value to avoid negative counts), then average intensities were taken over areas of different thicknesses. The intensity of the thinnest layer, which is consistent with the weakest HAADF signal, shows single-layer behaviour in the low-loss EELS spectrum. This is set to a value of one and all the other intensities calculated accordingly. It was found that for up to $\sim 5$ layers the intensity would increase as a multiple of the weakest signal, that is, as multiples of a monolayer.

Although the lattice is visible in the raw HAADF images, it is feasible to reduce the noise by applying a low-pass filter to the FFT image, which includes the radial frequencies right up to the lowest-order a-plane diffraction spots, as well as the a-plane ([100], $[010]$ and $[-110]$ ) reflections themselves (inset, Fig. 3). The chosen filter, which was also applied to the HAADF image in Fig. 2b, achieves a decrease in the high frequencies, but preserves the information of all direct space distances larger than the a-lattice plane spacing, in addition to enhancing the graphene lattice. In order to investigate the contribution of artefacts introduced into the inverse Fourier transform (that is, into the end image by the mask edges and the background noise) we subtracted a filtered Fourier transformed vacuum image from the filtered graphene Fourier space image, before we applied the inverse transform. However, this showed insignificant change.

\section{ELECTRON ENERGY-LOSS SPECTROSCOPY (EELS) INTENSITY}

As the analysed sample was extremely thin there was no case for the removal of plural scattering from the EELS data (the mean free path for scattering events in a $100 \mathrm{kV}$ machine for carbon is of the order of $100 \mathrm{~nm}$; ref. 23). To calculate the thickness of the sample, the area under the acquired spectrum between 10 and $40 \mathrm{eV}$ was integrated.

As a result of the background noise there was a small measured thickness in vacuum; this value was subtracted from all other measurements. The relative intensities were then calculated with respect to the thinnest layer (showing single-layer behaviour in the EELS signal), which was set to a value of 1 . The intensities for clean areas of the sample increased as multiples of the monolayer and were in good agreement with the measurements from the HAADF images for the same areas.

Received 16 July 2008; accepted 29 August 2008; published 28 September 2008.

References

1. Novoselov, K. S. et al. Two-dimensional gas of massless Dirac fermions in graphene. Nature 438, 197-200 (2005).

2. Zhang, Y., Tan, Y. W., Stormer, H. L. \& Kim, P. Experimental observation of the quantum Hall effect and Berry's phase in graphene. Nature 438, 201-204 (2005).

3. Geim, A. K. \& Novoselov, K. S. The rise of graphene. Nature Mater. 6, 183-191 (2007).

4. Schedin, F. et al. Detection of individual gas molecules by graphene sensors. Nature Mater. 6 , 652-655 (2007).

5. Novoselev, K. S. et al. Room temperature quantum Hall effect in graphene. Science 315,1379 (2007).

6. Meyer, J. C. et al. The structure of suspended graphene sheets. Nature 446, 60-63 (2007).

7. Fasolino, A., Los, J. H. \& Katsnelson, M. I. Intrinsic ripples in graphene. Nature Mater. 6, $858-861$ (2007)

8. Meyer, J. C. et al. Direct imaging of lattice atoms and topological defects in graphene membranes. Nano Lett. doi: $10.1021 / \mathrm{nl} 801386 \mathrm{~m}$ (2008).

9. Eberlein, T. et al. Plasmon spectroscopy of free-standing graphene films. Phys. Rev. B 77, $233406(2008)$

10. Stephan, O., Taverna, D., Kociak, M., Charbois, V. \& Henrard, L. Electron energy-loss spectrum of an electron passing near a locally anisotropic nanotube. Phys. Rev. B 66, 235419 (2002).

11. Kociak, M., Henrard, L., Stephan, O., Suenaga, K. \& Colliex, C. Plasmons in layered nanospheres and nanotubes investigated by spatially resolved electron energy-loss spectroscopy. Phys. Rev. B 61, 13936-13944 (2000)

12. Li, Z. Y. et al. Three-dimensional atomic-scale structure of size-selected gold nanoclusters. Nature $451,46-48$ (2008)

13. Filippi, M. \& Calliari, L. Measuring the energy of the graphitic $\pi+\sigma$ plasmon peak. Surf. Interface Anal. 38, 595-598 (2006).

14. Nair, R. R. et al. Fine structure constant defines visual transparency of graphene. Science 320, 1308 (2008).

15. Hahn, J. R. \& Kang, H. Vacancy and interstitial defects at graphite surfaces: Scanning tunnelling microscopic study of the structure, electronic property and yield for ion-induced defect creation. Phys. Rev. B 60, 6007-6017 (1999).

16. Bourelle, E., Konno, H. \& Inagaki, M. Structural defects created on natural graphite surface by slight treatment of oxygen plasma-STM observations. Carbon 37, 2041-2048 (1999).

17. Telling, R. H. \& Heggie, M. I. Radiation defects in graphite. Phil. Mag. 87, 4797-4846 (2007).

18. Krasheninnikov, A. V., Nordlund, K., Sirviö, M., Salonen, E. \& Keinonen, J. Formation of ion-irradiation-induced atomic-scale defects on walls of carbon nanotubes. Phys. Rev. B 63 , 245405 (2001).

19. Terrones, M. \& Terrones, H. The role of defects in graphitic structures. Fuller. Nanotub. Carbon Nanostruct. 4, 517-533 (1996)

20. Rotkin, S. V. \& Gogotsi, Y. Analysis of non-planar graphitic structures: From arched edge planes of graphite crystals to nanotubes. Mater. Res. Innovat. 5, 191-200 (2002).

21. Moriguchi, K. et al. Nano-tube-like surface structure in graphite particles and its formation mechanism: A role in anodes of lithium-ion secondary batteries. J. Appl. Phys. 88, 6369-6376 (2000).

22. Novoselov, K. S. et al. Electric field effect in atomically thin carbon films. Science 306, 666-669 (2004).

23. Egerton, R. F. Electron Energy Loss Spectroscopy in the Electron Microscope 2nd edn, 304 (Plenum, 1996).

Acknowledgements

The authors wish to thank the Engineering and Physical Sciences Research Council (EPSRC) for SuperSTEM funding under grant EP/D040396/1.

Author contributions

M.H.G., U.B., A.L.B. and A.K.G. conceived and designed the experiments. R.R.N. performed sample preparation. M.H.G., U.B. and A.L.B. carried out the experiments and performed data analysis.

P.W. performed the image simulations. M.H.G., U.B. and A.L.B. co-wrote the paper. All authors discussed the results and commented on the manuscript.

\section{Author information}

Reprints and permission information is available online at http://npg.nature.com/reprintsandpermissions/. Correspondence and requests for materials should be addressed to M.H.G. 